\title{
QUALITATIVE DIFFERENTIATION
}

\author{
BY
}

MICHAEL J. EVANS AND LEE LARSON

\begin{abstract}
Qualitative derivates and derivatives, as well as qualitative symmetric derivates and derivatives, are studied in the paper. Analogues of several results known for ordinary derivates and derivatives are obtained in the qualitative setting.
\end{abstract}

1. Introduction. The notions of qualitative limits, qualitative continuity, and qualitative derivatives were introduced by S. Marcus [13-15]. The purpose of the present paper is to examine qualitative differentiation and qualitative symmetric differentiation and, in particular, to present analogues of results known to hold for ordinary differentiation, symmetric differentiation, approximate differentiation, and approximate symmetric differentiation. Loosely speaking, qualitative differentiation and qualitative symmetric differentiation may be thought of as category analogues of approximate differentiation and approximate symmetric differentiation, where the set neglected near a point in the computation of difference quotients is of first category at the point in the former setting instead of density zero at the point as in the latter.

We state our definitions in $\$ 2$. In $\$ 3$ we examine qualitative derivatives and derivates. There we show that a qualitatively differentiable function on the real line is actually differentiable everywhere and obtain what may be viewed as qualitative analogues of the Denjoy-Young-Saks theorem [18]. In $\$ 4$ we consider qualitative symmetric derivatives and derivates. We show that with mild continuity restrictions on the primitive, a qualitative symmetric derivative must belong to Baire class one and actually be the symmetric derivative of a closely related function except at countably many points. A monotonicity theorem and related results are given.

2. Definitions and notation. Here we list the definitions of most of the concepts and terms employed in the sequel.

We denote the complement and closure of a subset $A$ of the real line $R$ by $A^{c}$ and $\bar{A}$, respectively, and if $A$ is Lebesgue measurable, $|A|$ denotes its measure. We use $A \backslash B$ to denote $A \cap B^{c}$. If $A^{c}$ is a first category set, we say $A$ is residual. Similarly, if $I \backslash A$ is a first category set for some interval $I$, we say $A$ is residual in $I$.

Received by the editors October 1, 1982. Presented at the Sixth Summer Symposium on Real Analysis, June 23, 1983. The symposium was sponsored by the Real Analysis Exchange and the University of Waterloo.

1980 Mathematics Subject Classification. Primary 26A24; Secondary 26A48. 
Let $f$ denote a real-valued function defined on $R$. The restriction of $f$ to a set $A$ is denoted by $f l_{A}$. The qualitative limit superior of $f$ at $x_{0}$ is defined by $\underset{x \rightarrow x_{0}}{q-\varlimsup_{x \rightarrow m}} f(x)=\inf \left\{y:\{x: f(x)>y\}\right.$ is first category in some neighborhood of $\left.x_{0}\right\}$, and the qualitative limit inferior of $f$ at $x_{0}$ by

$q-\underline{\lim } f(x)=\sup \left\{y:\{x: f(x)<y\}\right.$ is first category in some neighborhood of $\left.x_{0}\right\}$. $\overline{x \rightarrow}_{0}$

If $q-\varlimsup_{x \rightarrow x_{0}} f(x)=q-\underline{\lim }_{x \rightarrow x_{0}} f(x)$, the common value is called the qualitative limit of $f$ at $x_{0}$ and is denoted by $q-\lim _{x \rightarrow x_{0}} f(x)$.

Left and right qualitative limits superior and inferior are defined in the obvious manner.

In the event that $f\left(x_{0}\right)=q-\lim _{x \rightarrow x_{0}} f(x)$ we say that $f$ is qualitatively continuous at $x_{0}$. We also let

$$
C_{q}(f)=\{x: f \text { is qualitatively continuous at } x\},
$$

and as is customary

$$
C(f)=\{x: f \text { is continuous at } x\} .
$$

The upper right qualitative derivate of $f$ at $x_{0}$ is defined by

$$
Q^{+} f\left(x_{0}\right)=\underset{h \rightarrow 0^{+}}{q-\varlimsup_{0}} \frac{f\left(x_{0}+h\right)-f(x)}{h} .
$$

Then $Q^{-} f\left(x_{0}\right), Q_{+} f\left(x_{0}\right)$ and $Q_{-} f\left(x_{0}\right)$ are all defined in the obvious manner. In the event that all four of these derivates are equal, we call their common value the qualitative derivative (or, for brevity, the q-derivative) of $f$ at $x_{0}$ and denote it by $f_{q}^{\prime}\left(x_{0}\right)$, i.e.

$$
f_{q}^{\prime}\left(x_{0}\right)=\underset{h \rightarrow 0}{q-\lim _{h \rightarrow 0}} \frac{f\left(x_{0}+h\right)-f\left(x_{0}\right)}{h} .
$$

(Unless specifically stated otherwise, $\infty$ and $-\infty$ are acceptable values for $f_{q}^{\prime}\left(x_{0}\right)$ in this paper.) Right and left qualitative derivatives may be defined in the obvious manner.

The upper [lower] symmetric derivate of $f$ at $x_{0}$ is given by

$$
\begin{aligned}
D^{s} f\left(x_{0}\right)=\varlimsup_{h \rightarrow 0^{+}} \frac{f(x+h)-f(x-h)}{2 h}, & \\
& {\left[D_{s} f\left(x_{0}\right)=\lim _{h \rightarrow 0^{+}} \frac{f(x+h)-f(x-h)}{2 h}\right], }
\end{aligned}
$$

and if $D^{s} f\left(x_{0}\right)=D_{s} f\left(x_{0}\right)$, then $f$ is said to have a symmetric derivative $f^{s}\left(x_{0}\right)=$ $D^{s} f\left(x_{0}\right)$ at $x_{0}$.

Upper and lower qualitative symmetric derivates, $Q^{s} f\left(x_{0}\right)$ and $Q_{s} f\left(x_{0}\right)$, as well as the qualitative symmetric derivative (or q-symmetric derivative) $f_{q}^{s}\left(x_{0}\right)$ are defined in the natural manner. Again, infinite values are considered acceptable for all derivates and derivatives considered in this work. 
A moment's reflection on these definitions will convince the reader that for a continuous $f: R \rightarrow R, D^{+} f \equiv Q^{+} f, D^{-} f \equiv Q^{-} f$ and $D^{s} f \equiv Q^{s} f$, where $D^{+} f$ and $D^{-} f$ denote resp. the right and left upper Dini derivates. Thus qualitative differentiation of continuous functions is an entirely superfluous subject. Consequently, we shall be examining classes of functions and their derivatives where the functions are not $a$ priori continuous. We shall use the following abbreviations:

$\Sigma=\left\{f: C(f)\right.$ is dense and $f^{s}$ exists everywhere $\}$,

$\Sigma_{q}=\left\{f: C_{q}(f)\right.$ is dense and $f_{q}^{s}$ exists everywhere $\}$,

$\beta=\left\{f: f\right.$ has the property of Baire, i.e., there is a residual set $A$ such that $\left.f\right|_{A}$ is continuous (cf. [17]) $\}$, and

$\beta_{1}=\{f: f$ belongs to Baire class one $\}$.

Let $A \subseteq R$ and $x \in R$. We define the reflection of $A$ about $x$ to be the set

$$
R_{x}(A)=\{2 x-t: t \in A\} .
$$

If $x \leqslant \inf A, R_{x}(A)$ is called a left reflection, and if $x \geqslant \sup A, R_{x}(A)$ is called a right reflection. If $S \subseteq R$ and $I$ and $I^{\prime}$ are open intervals, we say that $I^{\prime}$ is a finite left [resp., right] reflection of $I$ in $S$ if there exists a finite collection $\left\{I_{1}, I_{2}, \ldots, I_{k}\right\}$ of open intervals and a corresponding subset $\left\{s_{1}, s_{2}, \ldots, s_{k-1}\right\}$ of $S$ such that $I=I_{1}$, $I^{\prime}=I_{k}$ and $I_{j+1}$ is the left [resp. right] reflection of $I_{j}$ about $s_{j}$ for $j=1,2, \ldots, k-1$.

E. P. Dolženko [6] has introduced the notion of porosity and it has recently found application in the study of differentiation; e.g., see $[1,2,21]$. The porosity of the set $A$ at the point $x \in R$ is defined to be $\varlimsup_{r \rightarrow 0^{+}} l(x, r, A) / r$ where $l(x, r, A)$ denotes the length of the largest open interval contained in the set $(x-r, x+r) \cap A^{c}$. The set $A$ is called porous if it has positive porosity at each of its points, and it is called $\sigma$-porous if it is a countable union of porous sets. Every $\sigma$-porous set is both of first category and measure zero, but the converse is false [20].

3. Qualitative derivatives and derivates. J. Leonard [12] proved that if $f$ is continuous on $R$ and has a right qualitative derivative at $x_{0}$, then it has an ordinary right derivative at $x_{0}$. An examination of his proof shows that only obvious minor changes are needed to show that if $f$ is right $q$-continuous on $R$ and has a right $q$-derivative at $x_{0}$, then $f$ has an ordinary right derivative at $x_{0}$. From this and the obvious fact that if $f$ has a finite right $q$-derivative at $x_{0}$, then $f$ must be right $q$-continuous at $x_{0}$, we can note the following.

REMARK 3.1. If $f: R \rightarrow R$ has a finite right $q$-derivative at each point in $R$, then $f$ has an ordinary right derivative at each point in $R$.

The finiteness of the right $q$-derivative is necessary here. For example, the characteristic function of the set of rational numbers has right $q$-derivative 0 at every irrational number and $-\infty$ at every rational number and yet has an ordinary right derivative nowhere. As seen from the first paragraph in this section, no such continuous function could exist, but Leonard [12] made the following observation.

REMARK 3.2. There is a $\beta_{1}$ Darboux function which possesses a rignt $q$-derivative everywhere on $R$ but which fails to have an ordinary right derivative at uncountably many points. 
Our first objective is to show how markedly different the situation is when two-sided derivatives (finite or infinite) are considered. Indeed, the following theorem shows that the notion of a $q$-differentiable function is a superfluous one, just as in the case with a $q$-continuous function, an observation made by Leonard [12].

THEOREM 3.1. If $f: R \rightarrow R$ has a q-derivative at each point of $R$, then $f$ has an ordinary derivative at each point of $R$.

Proof. Let $x_{0} \in R$ and suppose $f^{\prime}\left(x_{0}\right)$ does not exist.

Initially, consider the situation where $f_{q}^{\prime}\left(x_{0}\right)$ is finite. Through a translation and addition of a linear function we see that there is no loss of generality in assuming that $x_{0}=0=f(0)=f_{q}^{\prime}(0)$. Since $f^{\prime}(0)$ does not exist, there is a number $\alpha>0$ and a sequence $\left\{x_{n}\right\}$ converging to 0 such that $\left|f\left(x_{n}\right) / x_{n}\right|>\alpha$ for all $n$.

To become specific without losing generality, assume that $\left\{x_{n}\right\}$ decreases to 0 and that for each $n, f\left(x_{n}\right) / x_{n}>\alpha$. Since $f_{q}^{\prime}(0)=0$, there is a $\delta>0$ such that

$$
\{x: f(x) / x<\alpha\} \text { is residual in }(0, \delta) \text {. }
$$

Choose $m$ so large that $0<x_{m}<\delta$. We consider the two possibilities $f_{q}^{\prime}\left(x_{m}\right)>0$ and $f_{q}^{\prime}\left(x_{m}\right) \leqslant 0$ separately.

First, if $f_{q}^{\prime}\left(x_{m}\right)>0$, there is a $\delta^{\prime}$ such that $0<\delta^{\prime}<f\left(x_{m}\right) / \alpha-x_{m}$, and such that the set

$$
S=\left\{x \in\left(x_{m}, x_{m}+\delta^{\prime}\right): f(x)>f\left(x_{m}\right)\right\}
$$

is residual in $\left(x_{m}, x_{m}+\delta^{\prime}\right)$. But, if $x \in S$, then

$$
f(x) / x>f\left(x_{m}\right) / x>f\left(x_{m}\right) /\left(x_{m}+\delta^{\prime}\right)>\alpha,
$$

and this contradicts (1).

Next, if $f_{q}^{\prime}\left(x_{m}\right) \leqslant 0$, then since $f\left(x_{m}\right) / x_{m}>\alpha$, there is a $\delta^{\prime}$ such that $0<\delta^{\prime}<x_{m}$ and the set

$$
T=\left\{x \in\left(x_{m}-\delta^{\prime}, x_{m}\right): f(x)>\alpha x_{m}\right\}
$$

is residual in $\left(x_{m}-\delta^{\prime}, x_{m}\right)$, for otherwise we would have $Q^{-} f\left(x_{m}\right)=\infty>0$. For $x \in T$, we have $f(x) / x>\alpha x_{m} / x>\alpha$, which, again, contradicts (1).

Consequently, to this point we have shown that if $f_{q}^{\prime}\left(x_{0}\right)$ is finite, then $f^{\prime}\left(x_{0}\right)$ exists and, of course, $f^{\prime}\left(x_{0}\right)=f_{q}^{\prime}\left(x_{0}\right)$.

Now we turn to the situation where $f_{q}^{\prime}\left(x_{0}\right)$ is infinite. Without loss of generality, assume $x_{0}=0=f(0)$ and $f_{q}^{\prime}(0)=+\infty$. Since $f^{\prime}(0)$ does not exist, there is an $\alpha>0$ and a sequence $\left\{x_{n}\right\}$ converging to 0 , say from the right, such that $f\left(x_{n}\right) / x_{n}<\alpha$ for all $n$. Since $f_{q}^{\prime}(0)=+\infty$, there is a $\delta>0$ such that

$$
\{x: f(x) / x>\alpha\} \text { is residual in }(0, \delta) .
$$

Choose $m$ so large that $0<x_{m}<\delta$. Again, we consider the two possibilities $f_{q}^{\prime}\left(x_{m}\right)>0$ and $f_{q}^{\prime}\left(x_{m}\right) \leqslant 0$ separately.

First, if $f_{q}^{\prime}\left(x_{m}\right)>0$, there is a $\delta^{\prime}$ such that $0<\delta^{\prime}<\min \left\{x_{m}, x_{m}-f\left(x_{m}\right) / \alpha\right\}$ and the set

$$
U=\left\{x \in\left(x_{m}-\delta^{\prime}, x_{m}\right): f(x)<f\left(x_{m}\right)\right\}
$$


is residual in $\left(x_{m}-\delta^{\prime}, x_{m}\right)$. But, if $x \in U$, then

$$
\begin{aligned}
& f(x) / x<f\left(x_{m}\right) / x \leqslant \begin{cases}0 & \text { if } f\left(x_{m}\right) \leqslant 0, \\
\frac{f\left(x_{m}\right)}{x_{m}-\delta^{\prime}} & \text { if } f\left(x_{m}\right)>0,\end{cases} \\
& <\alpha,
\end{aligned}
$$

which contradicts (2).

Finally, if $f_{q}^{\prime}\left(x_{m}\right) \leqslant 0$, then there is a $\delta^{\prime}>0$ such that the set

$$
V=\left\{x \in\left(x_{m}, x_{m}+\delta^{\prime}\right): f(x)-f\left(x_{m}\right)<\alpha\left(x-x_{m}\right)\right\}
$$

is residual in $\left(x_{m}, x_{m}+\delta^{\prime}\right)$. However, for $x \in V$

$$
\begin{aligned}
\frac{f(x)}{x} & =\frac{f(x)-f\left(x_{m}\right)}{x-x_{m}} \cdot \frac{\left(x-x_{m}\right)}{x}+\frac{f\left(x_{m}\right)}{x_{m}} \cdot \frac{x_{m}}{x} \\
& <\alpha \cdot \frac{x-x_{m}}{x}+\alpha \cdot \frac{x_{m}}{x}=\alpha,
\end{aligned}
$$

again contradicting (2).

Consequently, if $f_{q}^{\prime}\left(x_{0}\right)=+\infty, f^{\prime}\left(x_{0}\right)=+\infty$ and the theorem is proved.

Next we turn our attention to the relationships between the four qualitative derivates, looking for what may be viewed as analogues of the Denjoy-Young-Saks theorem for Dini derivates [18]. Recall that in the case of approximate derivates, the structure of such a theorem is simpler in the case of a measurable function [4 or 8] than it is in the case of an arbitrary function [5]. We shall see that a similar situation holds for qualitative derivates, with functions having the Baire property playing the role of measurable functions. We begin by noting a fairly obvious reformulation of the definition of the class $\beta$ which we shall frequently use in the remainder of this paper. For completeness, we include its proof.

THEOREM 3.2. For $f: R \rightarrow R$ the following are equivalent:

(a) $C_{q}(f)$ is dense;

(b) $C_{q}(f)$ is residual; and

(c) $f \in \beta$.

Proof. First, we make the observation that

$$
\left\{x: f(x)>q-\varlimsup_{t \rightarrow x} f(t)\right\} \text { is a first category set. }
$$

To see this, suppose that this is not the case. Then, for some rational number $\alpha$, the set

$$
S_{\alpha}=\left\{x: f(x)>\alpha>q-\varlimsup_{t \rightarrow x} f(t)\right\}
$$

would be of second category and, hence, of second category in each subinterval of some open interval $I$. Let $x_{0} \in S_{\alpha} \cap I$. Since $\{x: f(x)>\alpha\}$ is of second category in each neighborhood of $x_{0}$, we would have $q$ - $\varlimsup_{t \rightarrow x_{0}} f(t) \geqslant \alpha$, contradicting $x_{0} \in S_{\alpha}$. Hence, (1) holds. 
Similarly,

$$
\{x: f(x)<q-\underset{t \rightarrow x}{\lim } f(t)\} \text { is a first category set. }
$$

Because of (1) and (2), in order to show that (a) implies (b), we need only show that if (a) holds, then the set

$$
A=\left\{x: q-\varlimsup_{t \rightarrow x} f(t)>q-\varliminf_{t \rightarrow x} f(t)\right\}
$$

is a first category set. For each natural number $n$, let

$$
A_{n}=\left\{x: q-\varlimsup_{t \rightarrow x} f(t)-q-\varlimsup_{t \rightarrow x}^{\lim } f(t) \geqslant 1 / n\right\} .
$$

Then $A=\cup_{n} A_{n}$. Each $A_{n}$ is easily seen to be closed and since $C_{q}(f)$ is dense each $A_{n}$ must be nowhere dense. Consequently, $A$ is a first category set and $C_{q}(f)$ is residual.

Next, assume that $C_{q}(f)$ is residual. We shall show that $f \in \beta$ by showing that $\left.f\right|_{C_{q}(f)}$ is continuous. Let $x_{0} \in C_{q}(f)$ and $\varepsilon>0$. There is a $\delta>0$ and a residual subset $S$ of $\left(x_{0}-\delta, x_{0}+\delta\right)$ such that

$$
\left|f\left(x_{0}\right)-f(y)\right|<\frac{\varepsilon}{2} \quad \text { for } y \in S .
$$

Suppose $t \in C_{q}(f)$ and $\left|t-x_{0}\right|<\delta$. Then there is a neighborhood $U$ of $t$ with $U \subseteq\left(x_{0}-\delta, x_{0}+\delta\right)$ and a residual subset $T$ of $U$ such that

$$
|f(t)-f(y)|<\frac{\varepsilon}{2} \quad \text { for } y \in T \text {. }
$$

Since $S \cap T \neq \varnothing,(3)$ and (4) yield $|f(t)-f(x)|<\varepsilon$ and hence $\left.f\right|_{C_{q}(f)}$ is continuous at $x_{0}$.

That (c) implies (a) is immediate.

C. J. Neugebauer [16] showed that if $f: R \rightarrow R$ is continuous, then $D^{-} f(x)$ $=D^{+} f(x)$ and $D_{-} f(x)=D_{+} f(x)$ for all $x$ in a residual set. The next result is the qualitative analogue, which yields Neugebauer's result in the event that $f$ is continuous.

THEOREM 3.3. If $f: R \rightarrow R$ belongs to $\beta$, then

$$
Q^{-} f(x)=Q^{+} f(x) \text { and } Q_{-} f(x)=Q_{+} f(x)
$$

for all $x$ in a residual set.

Proof. We shall show that the set $A=\left\{x: Q^{-} f(x)<Q^{+} f(x)\right\}$ is a first category set. For each rational number $r$, let

$$
A_{r}=\left\{x: Q^{-} f(x)<r<Q^{+} f(x)\right\} .
$$

Since $A=\cup A_{r}$, it suffices to show each $A_{r}$ first category; indeed, only $A_{0}$ need be shown to be first category since $f(x)$ could be replaced by $f(x)-r x$.

For each natural number $n$, set

$$
A_{0 n}=\left\{x \in A_{0}:\{h \in(0,1 / n): f(x-h)>f(x)\} \text { residual in }(0,1 / n)\right\} \text {. }
$$


Since $A_{0} \subseteq \cup_{n} A_{0 n}$, we need only show each $A_{0 n}$ first category. Suppose $A_{0 n}$ is second category. Then $A_{0 n}$ is second category in every subinterval of some interval $I$ of length less than $1 / n$.

Let $x_{0} \in A_{0} \cap I \cap C_{q}(f)$. Since $x_{0} \in A_{0}$, the set

$$
E=\left\{x \in I: x>x_{0} \text { and } f(x)>f\left(x_{0}\right)\right\}
$$

is second category in $I$. Let $x_{1} \in E \cap C_{q}(f)$. Then there is a neighborhood $J \subseteq I$ of $x_{1}$ such that $x_{0} \notin J$ and such that the set $S=\left\{x \in J: f(x)>f\left(x_{0}\right)\right\}$ is residual in $J$. Since $A_{0 n}$ is second category in $J$, there is a point $x_{2} \in A_{0 n} \cap S$. Since $x_{2} \in S$, we have

$$
f\left(x_{2}\right)>f\left(x_{0}\right)
$$

However, since $x_{2} \in A_{0 n}$ and $x_{0} \in C_{q}(f)$, we must have

$$
f\left(x_{0}\right) \geqslant f\left(x_{2}\right) \text {. }
$$

The contradiction of (1) and (2) completes the proof.

Notice that the previous theorem does not hold for arbitrary functions. For example, let $S$ be a set with the property that both it and its complement are of second category in every interval. Then the characteristic function of $S$ does not satisfy the conclusion of Theorem 3.3. The next result, however, does show a relationship between the qualitative derivates of an arbitrary function. It is a generalization to qualitative derivates of a theorem on ordinary derivates due to Belna, Cargo, Evans, and Humke [1] and, independently, Zajiček [21].

THEOREM 3.4. If $f: R \rightarrow R$ is arbitrary, then $R=A \cup B \cup C \cup D$ where

$$
\begin{aligned}
& A=\left\{x: Q^{-} f(x)=Q^{+} f(x) \text { and } Q_{-} f(x)=Q_{+} f(x)\right\}, \\
& B=\left\{x:-\infty=Q_{-} f(x) \leqslant Q_{+} f(x) \leqslant Q^{-} f(x) \leqslant Q^{+} f(x)=\infty\right\}, \\
& C=\left\{x:-\infty=Q_{+} f(x) \leqslant Q_{-} f(x) \leqslant Q^{+} f(x) \leqslant Q^{-} f(x)=\infty\right\},
\end{aligned}
$$

and $D$ is a first category set.

Proof. Define two extended real-valued functions, $u_{f}$ and $l_{f}$, by

$$
u_{f}(x)=q-\varlimsup_{t \rightarrow x} f(t) \quad \text { and } \quad l_{f}(x)=q-\underset{t \rightarrow x}{\lim } f(t)
$$

and let

$$
U_{f}=\left\{x: u_{f}(x)=\infty\right\} \text { and } L_{f}=\left\{x: l_{f}(x)=-\infty\right\} .
$$

It is apparent from their definitions that $u_{f}$ is upper semicontinuous and $l_{f}$ is lower semicontinuous; so both $u_{f}$ and $l_{f}$ are in $\beta_{1}$. This implies that the set of points at which both $u_{f}$ and $l_{f}$ are continuous (as extended real-valued functions) is residual. As was shown in the beginning of the proof of Theorem 3.2 the inequalities

$$
u_{f}(x)>f(x)>l_{f}(x)
$$

are true on a residual set. This implies that the set of all points at which (1) is true and both $u_{f}$ and $l_{f}$ are continuous is residual. Call this set $E$. It suffices to show that there exists a first category set $F$ such that $(E \backslash F) \subseteq(A \cup B \cup C)$. 
To do this, we first note that the semicontinuity of $u_{f}$ and $l_{f}$ guarantees that both $U_{f}$ and $L_{f}$ are closed. Let $I_{1}, I_{2}, \ldots$, be the components of $\left(U_{f}\right)^{c}$. For a fixed $n, u_{f \mid I_{n} \cap E}$ is continuous. Thus, $u_{f \mid I_{n}}$ satisfies the conditions of Theorem 3.3. Similarly, if $J_{1}, J_{2}, \ldots$, are the components of $\left(L_{f}\right)^{c}$, then $l_{f \mid J_{m}}$ satisfies the conditions of Theorem 3.3. Consequently, if we let $H$ denote the set of all $x$ in $\left(U_{f}\right)^{c}$ at which at least one of the equalities

$$
Q^{-} u_{f}(x)=Q^{+} u_{f}(x), \quad Q_{-} u_{f}(x)=Q_{+} u_{f}(x),
$$

fails to hold, and $K$ denote the set of all $x \in\left(L_{f}\right)^{c}$ at which at least one of the equalities

$$
Q^{-} l_{f}(x)=Q^{+} l_{f}(x), \quad Q_{-} l_{f}(x)=Q_{+} l_{f}(x),
$$

is false and let $F=H \cup K$, then $F$ is clearly a first category set.

Now let $x \in E \backslash F$. In light of (1) there are three cases to consider.

Case 1. Suppose $u_{f}(x)=f(x)$. Then, of course, $x \in\left(U_{f}\right)^{c}, u_{f}$ is finite valued on a neighborhood of $x$, and $u_{f} \geqslant f$ on a residual subset of that neighborhood. Consequently,

$$
Q^{+} u_{f}(x) \geqslant Q^{+} f(x) .
$$

Suppose strict inequality holds in (4). To be specific, there is no generality lost with the assumption that

$$
Q^{+} u_{f}(x)>0>Q^{+} f(x) .
$$

This implies the existence of an $\varepsilon>0$ such that $\{t \in(0, \varepsilon): f(x)>f(t)\}$ is residual in $(0, \varepsilon)$. But the definition of $u_{f}$ then yields

$$
\left\{t \in(0, \varepsilon): u_{f}(x)=f(x)>u_{f}(t)\right\}=(0, \varepsilon),
$$

which implies $D^{+} u_{f}(x) \leqslant 0$, contradicting (5). Hence

$$
Q^{+} u_{f}(x)=Q^{+} f(x) \text {. }
$$

A similar argument yields

$$
Q_{-} u_{f}(x)=Q_{-} f(x)
$$

Case 2. Suppose $l_{f}(x)=f(x)$. In this case, using $-f$ in the argument of Case 1 yields $x \in\left(L_{f}\right)^{c}$ and

$$
Q_{+} l_{f}(x)=Q_{+} f(x) \text { and } Q^{-} l_{f}(x)=Q^{-} f(x) .
$$

At this point we note that if Cases 1 and 2 both apply at $x$, then since $x \in\left[\left(U_{f}\right)^{c} \cap\left(L_{f}\right)^{c}\right] \backslash F$, we see that all the equalities (2), (3), (6), (7) and (8) hold and, hence, $x \in A$. On the other hand, if only Case 1 applies at $x$, then $u_{f}(x)=f(x)$ $>l_{f}(x)$. Then equalities (2), (6) and (7) hold. Furthermore, we clearly have from the continuity of $l_{f}$ at $x$ and the fact that $f(x)>l_{f}(x)$ that

$$
-\infty=Q_{+} f(x) \text { and } Q^{-} f(x)=\infty .
$$

Applying (2), (6) and (7) we obtain

$$
-\infty=Q_{+} f(x) \leqslant Q_{-} f(x)=Q_{-} u_{f}(x) \leqslant Q^{-} u_{f}(x)=Q^{+} f(x) \leqslant Q^{-} f(x)=\infty,
$$

and hence $x \in C$. Similarly, if only Case 2 applies at $x$, then $x \in B$. 
Case 3. Suppose $l_{f}(x)<f(x)<u_{f}(x)$. When this occurs, it is clear from the continuity of $l_{f}$ and $u_{f}$ at $x$ that

$$
Q_{+} f(x)=Q_{-} f(x)=-\infty \text { and } Q^{+} f(x)=Q^{-} f(x)=\infty
$$

so that $x \in A$, completing the proof.

COROllary 3.1. If $f: R \rightarrow R$ is arbitrary and $P$ denotes the set of points at which $f$ has a finite left or right qualitative derivative, then $\left\{x \in P: f_{q}^{\prime}(x)\right.$ does not exist $\}$ is of first category.

In the version of Theorem 3.4 for ordinary derivates (Theorem 2 in [1] or Theorem 1 in [21]) the exceptional set $D$ is $\sigma$-porous. That this need not be the case in the qualitative setting, even for functions in $\beta$, is seen by considering the characteristic function $f$ of a first category set $S$ whose complement has measure zero. (For the existence of such a set see [17].) For $x \in S$ we have

$$
Q^{-} f(x)=Q_{-} f(x)=\infty \text { and } Q^{+} f(x)=Q_{+} f(x)=-\infty .
$$

However, we can obtain the following result.

THEOREM 3.5. If $f: R \rightarrow R$ is arbitrary and if the sets $A, B$ and $C$ are as in Theorem 3.4, then $C_{q}(f) \backslash(A \cup B \cup C)$ is o-porous.

Proof. We find it convenient to split the proof into two cases. First, we consider the case where $f \in \beta$; then we take up an arbitrary $f$.

If $f \in \beta$, we let $h: C_{q}(f) \rightarrow R$ denote the restriction of $f$ to $C_{q}(f)$. Since $C_{q}(f)$ is residual, it is an easy matter to see that for $x \in C_{q}(f)$, each ordinary derivate for $h$ is equal to the corresponding qualitative derivate for $f$; e.g., $D^{+} h(x)=Q^{+} f(x)$. Hence, an application of Theorem 2 in [1] to $h$ produces the required conclusion.

Now suppose that $f: R \rightarrow R$ is arbitrary. Let $u_{f}, l_{f}, U_{f}$ and $L_{f}$ have the same meanings as in the proof of Theorem 3.4. As observed there, on each component interval of $\left(U_{f}\right)^{c}, u_{f}$ is a finite-valued function possessing the Baire property and hence the first case of the present proof may be applied there to $u_{f}$. Noting that $C_{q}(f) \subseteq\left(U_{f}\right)^{c}$ and that on each component interval $I$ of $\left(U_{f}\right)^{c}$ we have $\left(C_{q}(f) \cap I\right) \subseteq$ $C_{q}\left(u_{f \mid}\right)$, it follows that

$$
C_{q}(f) \backslash\left(A_{u} \cup B_{u} \cup C_{u}\right) \text { is } \sigma \text {-porous, }
$$

where

$$
\begin{aligned}
& A_{u}=\left\{x \in\left(U_{f}\right)^{c}: Q^{-} u_{f}(x)=Q^{+} u_{f}(x) \text { and } Q_{-} u_{f}(x)=Q_{+} u_{f}(x)\right\}, \\
& B_{u}=\left\{x \in\left(U_{f}\right)^{c}:-\infty=Q_{-} u_{f}(x) \leqslant Q_{+} u_{f}(x) \leqslant Q^{-} u_{f}(x) \leqslant Q^{+} u_{f}(x)=\infty\right\}
\end{aligned}
$$
and

$$
C_{u}=\left\{x \in\left(U_{f}\right)^{c}:-\infty=Q_{+} u_{f}(x) \leqslant Q_{-} u_{f}(x) \leqslant Q^{+} u_{f}(x) \leqslant Q^{-} u_{f}(x)=\infty\right\} .
$$

Similarly, examining $l_{f}$ on $\left(L_{f}\right)^{c}$ we see that

$$
C_{q}(f) \backslash\left(A_{l} \cup B_{l} \cup C_{l}\right) \text { is } \sigma \text {-porous, }
$$

where $A_{l}, B_{l}$ and $C_{l}$ are the subsets of $\left(L_{f}\right)^{c}$ defined in the obvious manner.

Recall that for $x \in C_{q}(f)$ we have

$$
\begin{gathered}
Q^{+} u_{f}(x)=Q^{+} f(x), \quad Q_{-} u_{f}(x)=Q_{-} f(x), \\
Q_{+} l_{f}(x)=Q_{+} f(x) \text { and } Q^{-} l_{f}(x)=Q^{-} f(x) .
\end{gathered}
$$


(See (6)-(8) in the proof of Theorem 3.4.) Utilizing these four equalities (3), it is a routine matter to show that

$$
\begin{array}{lll}
C_{q}(f) \cap A_{u} \cap A_{l} \subseteq A, & C_{q}(f) \cap A_{u} \cap B_{l} \subseteq A, & C_{q}(f) \cap A_{u} \cap C_{l} \subseteq C, \\
C_{q}(f) \cap B_{u} \cap A_{l} \subseteq B, & C_{q}(f) \cap B_{u} \cap B_{l} \subseteq B, & C_{q}(f) \cap B_{u} \cap C_{l} \subseteq A, \\
C_{q}(f) \cap C_{u} \cap B_{l} \subseteq A, & C_{q}(f) \cap C_{u} \cap C_{l} \subseteq C, & C_{q}(f) \cap C_{u} \cap A_{l} \subseteq A .
\end{array}
$$

These nine inclusions together with (1) and (2) complete the proof.

The final result of this section, which is a direct consequence of the previous theorem, is the qualitative version of Theorem 1 in [7].

COROLlary 3.2. If $f: R \rightarrow R$ is monotone on a residual set $S$, then

$$
S \backslash\left\{x: Q^{-} f(x)=Q^{+} f(x) \text { and } Q_{-} f(x)=Q_{+} f(x)\right\}
$$

is a $\sigma$-porous set.

4. Qualitative symmetric derivatives and derivates. In this section, we shall deal with $q$-symmetric differentiation. This topic holds somewhat more innate interest than that of $q$-differentiation in that a $q$-symmetrically differentiable function need not be symmetrically differentiable anywhere.

REMARK 4.1. There is a function $f: R \rightarrow R$ that possesses a $q$-symmetric derivative of constant value positive one at each point in $R$ and an approximate symmetric derivative of constant value negative one at each point in $R$. Consequently, $f$ has a symmetric derivative nowhere.

To construct such a function recall that the real line may be expressed as the disjoint union of a first category set $A$ and a measure zero set $B$; e.g., see [17]. Then let $f(x)=-x$ if $x \in A$ and $f(x)=x$ if $x \in B$. Clearly, $f_{q}^{s}(x)=1$ for every $x$. Now, the approximate symmetric derivative of $f$ at $x$ is defined as the limit as $h$ approaches 0 through a set having density 1 at 0 of the symmetric difference quotient

$$
[f(x+h)-f(x-h)] / 2 h,
$$

provided this limit exists, cf. $[10,22]$. But since $A$ has density 1 at each point of $R$, we clearly have the approximate symmetric derivative existing with value negative one everywhere. However, if a function has a symmetric derivative at a point, then it must have both a qualitative symmetric and an approximate symmetric derivative at that point and all three values must be identical.

Next, we observe that a function $f: R \rightarrow R$ can have a $q$-symmetric derivative at each point of a second category set $S$ and yet have a $q$-derivative at no point of $S$. To obtain such a function, note that there is an additive group $S$ of real numbers having the property that both it and its complement are of second category in every interval. Such a group is constructed in [3]. If we let $f$ denote the characteristic function of $S$, then $f$ has in fact symmetric derivative 0 at each point of $S$, but has a $q$-derivative nowhere.

It appears, therefore, especially in light of analogous results concerning ordinary symmetric differentiation [2, 11], that we need to add some sort of continuity hypothesis to $f$ if we wish to establish that the existence of $f_{q}^{s}$ on a "large" set $S$ implies the existence of $f_{q}^{\prime}$ on a "large" subset of $S$. The condition which yielded this 
type of result in the case of ordinary symmetric differentiation [2] was that of requiring $C(f)$ to be dense. It seems natural, therefore, to expect positive results in the qualitative setting by requiring $C_{q}(f)$ to be dense and, as shown in Theorem 3.2, this is equivalent to requiring that $f \in \beta$.

LEMMA 4.1. If $f: R \rightarrow R$ is in $\beta$ and if

$S=\left\{x \in C_{q}(f):\right.$ there is a $\delta>0$

such that $\{h \in(0, \delta): f(x-h) \leqslant f(x+h)\}$ is residual in $(0, \delta)\}$,

and

$I=\left\{x \in C_{q}(f)\right.$ : there is $a \delta>0$

such that $\{h \in(0, \delta): f(x-h) \leqslant f(x) \leqslant f(x+h)\}$ is residual in $(0, \delta)\}$,

then $S \backslash I$ is a $\sigma$-porous set.

Proof. First notice that $I=I^{*}$, where

$I^{*}=\left\{x \in C_{q}(f)\right.$ : there is a $\delta>0$ and a set $T_{x}$ residual in $(0, \delta)$ such that if $h, k \in T_{x}$, then $\left.f(x-h) \leqslant f(x+k)\right\}$.

For each natural number $n$ let

$S_{n}=\left\{x \in C_{q}(f):\{h \in(0,1 / n): f(x-h) \leqslant f(x+h)\}\right.$ is residual in $\left.(0,1 / n)\right\}$.

Then $S=\cup S_{n}$ and it suffices to show that each $S_{n} \backslash I^{*}$ is porous. We shall actually show that $S_{n} \backslash I^{*}$ has porosity $\geqslant 1 / 2$ at each of its points.

Suppose $x_{0} \in S_{n} \backslash I^{*}$ and that the porosity of $S_{n} \backslash I^{*}$ at $x_{0}$ is less than $1 / 2$. For simplicity of notation and without loss of generality, assume $x_{0}=0$. Since the porosity of $S_{n} \backslash I^{*}$ is $<1 / 2$, there is a $0<\delta<1 / n$ such that $l\left(0, r, S_{n} \backslash I^{*}\right) / r<1 / 2$ for all $0<r \leqslant \delta$.

Since $0 \notin I^{*}$, there is a number $\alpha$, a set $A$ of second category in $(-\delta, 0)$ and a set $B$ of second category in $(0, \delta)$ such that for $a \in A$ and $b \in B, f(a)>\alpha>f(b)$. Since $C_{q}(f)$ is residual, $C_{q}(f) \cap A \neq \varnothing$ and $C_{q}(f) \cap B \neq \varnothing$ and, consequently, we may find open intervals $H \subseteq(-\delta, 0)$ and $K \subseteq(0, \delta)$ such that

$$
\{x: f(x)>\alpha\} \text { is residual in } H
$$

and

$$
\{x: f(x)<\alpha\} \text { is residual in } K .
$$

Now, according to Lemma 1 in [2], $H$ has a finite right reflection in $S_{n}$, call it $H^{*}$, such that $0 \in H^{*}$. Furthermore, $K$ has a finite left reflection in $S_{n}$, call it $K^{*}$, such that $0 \in K^{*}$. Since we reflect about points in $S_{n}$, it is clear from (1) and (2) that

$$
\{x: f(x)>\alpha\} \text { is residual in } H^{*}
$$

and

$$
\{x: f(x)<\alpha\} \text { is residual in } K^{*} .
$$

However, (3) and (4) now present us with an impossible situation in the nonempty open interval $H^{*} \cap K^{*}$. This contradiction completes the proof. 
THEOREM 4.1. If $f: R \rightarrow R$ is in $\beta$ and if $T$ denotes the set of points $x$ at which both

(i) $Q_{s} f(x)=\min \left\{Q_{-} f(x), Q_{+} f(x)\right\}$ and

(ii) $Q^{s} f(x)=\max \left\{Q^{-} f(x), Q^{+} f(x)\right\}$,

then $C_{q}(f) \backslash T$ is $\sigma$-porous.

Proof. We shall treat (i) only here; (ii) can then be handled by considering $-f$. We proceed along the lines of the proof of Theorem 2 in [2].

First, note that at each point $x \in R$ we have

$$
Q_{s} f(x) \geqslant \min \left\{Q_{-} f(x), Q_{+} f(x)\right\} \text {. }
$$

Consequently, we need only show that the set of points in $C_{q}(f)$ at which this inequality is strict is a $\sigma$-porous set. To this end, for each rational number $\alpha$ set

$$
N(f, \alpha)=\left\{x \in C_{q}(f): Q_{s} f(x)>\alpha>\min \left\{Q_{-} f(x), Q_{+} f(x)\right\}\right\} .
$$

It then suffices to show that each set $N(f, \alpha)$ is $\sigma$-porous. Since $N(f, \alpha)=N(g, 0)$ for the function $g(x)=f(x)-\alpha x$, it is sufficient to show that $N(f, 0)$ is $\sigma$-porous. This, however, is an immediate consequence of Lemma 4.1 since $N(f, 0) \subseteq S \backslash I$.

Corollary 4.1. If $f: R \rightarrow R$ is in $\beta$, then the set of points in $C_{q}(f)$ at which $f$ has a $q$-symmetric derivative, but not a q-derivative, is a $\sigma$-porous set.

A weaker but possibly equally applicable statement is the following:

COROLlaRY 4.2. If $f \in \Sigma_{q}$, then $f_{q}^{\prime}$ exists at a residual set of points.

It was shown in [10] that the approximate symmetric derivative of a measurable function belongs to Baire class one. The analogue in the qualitative setting is the following:

THEOREM 4.2. If $f \in \Sigma_{q}$, then $f_{q}^{s} \in \beta_{1}$.

Proof. Let $f \in \Sigma_{q}$ and let $\alpha>0$ and $r \in R$. We shall first show that the two sets and

$A_{f}(\alpha, r)=\{x:\{t \in(0, \alpha): f(x+t)-f(x-t)>2 t r\}$ is second category $\}$

$B_{f}(\alpha, r)=\{x:\{t \in(0, \alpha): f(x+t)-f(x-t)<2 t r\}$ is second category $\}$ are open.

Since $B_{f}(\alpha, r)=A_{-f}(\alpha,-r)$, it suffices to consider $A_{f}(\alpha, r)$ only. Also, since $A_{f}(\alpha, r)=A_{g}(\alpha, 0)$, where $g(x)=f(x)-r x$, it suffices to show $A_{f}(\alpha, 0)$ open.

Suppose $x_{0} \in A_{f}(\alpha, 0)$. For notational simplicity and without loss of generality, assume $x_{0}=0$. Let $T=\{t \in(0, \alpha): f(t)-f(-t)>0\}$. Then $T$ is second category. Since $f$ has the Baire property, there is a residual set $S$ such that $\left.f\right|_{S}$ is continuous. Since $R_{0}(S)$ is also residual, we may as well assume that $R_{0}(S)=S$. Let $t_{0} \in T \cap S$ and let $\varepsilon=f\left(t_{0}\right)-f\left(-t_{0}\right)>0$. Let $I$ be an open interval containing $t_{0}$ with $|I|<\left(\alpha-t_{0}\right) / 2$ such that for $t \in I \cap S$ we have

$$
\left|f(t)-f\left(t_{0}\right)\right|<\varepsilon / 2 \text { and }\left|f(-t)-f\left(-t_{0}\right)\right|<\varepsilon / 2 .
$$

Now, let $x \in(-|I| / 2,|I| / 2)$. Clearly,

$$
R_{x}(I) \cap R_{0}(I) \neq \varnothing
$$


and hence $R_{x}(I \cap S) \cap R_{0}(I \cap S)$ is of second category. Also, note that $\{h: x+h$ $\in I \cap S\} \subseteq(0, \alpha)$. Consequently,

$$
\left\{h \in(0, \alpha): x+h \in I \cap S \text { and } x-h \in R_{0}(I \cap S)\right\}
$$

is of second category. Let $h$ belong to this set. From (1) we have

$$
f(x+h)-f(x-h)>f\left(t_{0}\right)-\varepsilon / 2-f\left(-t_{0}\right)-\varepsilon / 2=0 .
$$

Therefore, $\{h \in(0, \alpha): f(x+h)-f(x-h)>0\}$ is second category, $x \in A_{f}(\alpha, 0)$, and $A_{f}(\alpha, 0)$ is, consequently, open.

Since the $q$-limit as $h$ approaches zero from the right of $(f(x+h)-f(x-h)) / 2 h$ is $f_{q}^{s}(x)$ for each $x \in R$, it is easy to see that for each $\gamma \in R$,

$$
\left\{x: f_{q}^{s}(x) \geqslant \gamma\right\}=\bigcap_{m=1}^{\infty} \bigcap_{n=1}^{\infty} A_{f}(1 / n, \gamma-1 / m)
$$

and

$$
\left\{x: f_{q}^{s}(x) \leqslant \gamma\right\}=\bigcap_{m=1}^{\infty} \bigcap_{n=1}^{\infty} B_{f}(1 / n, \gamma+1 / m) .
$$

Each of these sets is a $G_{\delta}$ and, consequently, $f_{q}^{s} \in \beta_{1}$.

Most of the remaining results in this section parallel those in the case of symmetric differentiation as developed by Larson in [11]. We begin by identifying what might be thought of as the set of "bad" points of a $q$-symmetrically differentiable function.

DeFinition 4.1. For $f \in \Sigma_{q}$, set

$$
M_{f}=\left\{x:\left|q-\varlimsup_{t \rightarrow x} f(t)\right|=\infty\right\} .
$$

THEOREM 4.3. If $f \in \Sigma_{q}$, then $\bar{M}_{f}$ is countable.

Proof. We shall show that the set

$$
A=\left\{x: q-\varlimsup_{t \rightarrow x} f(t)=+\infty\right\}
$$

is closed and countable. That $A$ is closed is obvious. So we may write $A=P \cup N$ where $P$ is perfect and $N$ is countable. Suppose $P$ is not empty. Choose an arbitrary interval $(\alpha, \beta)$ contiguous to $P$. Since $P$ is not empty, one of the numbers $\alpha$ or $\beta$ must be finite. Suppose $\alpha$ is finite. Choose a sequence $\left\{\alpha_{n}\right\} \subseteq P$ such that $\alpha_{n}$ increases to $\alpha$ and $R_{\alpha}\left(\left\{\alpha_{n}\right\}\right) \cap A=\varnothing$.

Now, let $\delta>0$ and choose $n$ so large that $\alpha_{n} \in(\alpha-\delta, \alpha)$. Since $R_{\alpha}\left(\alpha_{n}\right) \notin A$ we have

$$
\underset{t \rightarrow R_{\alpha}\left(\alpha_{n}\right)}{q-\varlimsup_{S}} f(t)<+\infty .
$$

Consequently, there is a set $S_{n}$ which is second category in $(\alpha-\delta, \alpha)$ such that

$$
f(x)-f\left(R_{\alpha}(x)\right)>n \text { for } x \in S_{n} .
$$

And now, since $f_{q}^{s}(\alpha)$ was assumed to exist and since $\delta$ is arbitrary, we see that $f_{q}^{s}(\alpha)=-\infty$. Similarly, $f_{q}^{s}(\beta)=+\infty$ if $\beta<\infty$. 
From Theorem 4.3 we have that both $\left\{x: f_{q}^{s}(x)=+\infty\right\}$ and $\left\{x: f_{q}^{s}(x)=-\infty\right\}$ are $G_{\delta}$ sets. Consequently, we have the situation of two disjoint, dense $G_{\delta}$ sets in the Baire space $P$. This is impossible. Hence $P=\varnothing$ and $A$ is countable.

By considering $-f$ instead of $f$ above, we see that $B=\left\{x: q-\lim _{t \rightarrow x} f(t)=-\infty\right\}$ is also closed and countable. Since $M_{f} \subseteq A \cup B$, the theorem follows.

Now, if $f \in \Sigma_{q}$ there obviously are infinitely many $g \in \Sigma_{q}$, such that $f_{q}^{s} \equiv g_{q}^{s}$. We seek such a $g$ that will agree with $f$ at each $x \in C_{q}(f)$ and be as "nice" as possible in some sense.

Definition 4.2. For $g \in \Sigma_{q}$, define

$$
\mu_{f}(x)= \begin{cases}q-\overline{\lim } f(t) & \text { if } x \notin M_{f}, \\ f(x) & \text { if } x \in M_{f} .\end{cases}
$$

THEOREM 4.4. If $f \in \Sigma_{q}$, then

(a) $C_{q}(f) \subseteq C\left(\mu_{f}\right)$ and $f(x)=\mu_{f}(x)$ when $x \in C_{q}(f)$;

(b) $\mu_{f} \in \Sigma_{q}$ and $\left(\mu_{f}\right)_{q}^{s} \equiv f_{q}^{s}$;

(c) $\mu_{f}(x)=\varlimsup_{t \rightarrow x} \mu_{f}(t)$ on $\left(\bar{M}_{f}\right)^{c}$;

(d) $\mu_{f} \in \beta_{1}$; and

(e) $\left(\mu_{f}\right)^{s}$ exists and equals $f_{q}^{s}$ on $\left(\bar{M}_{f}\right)^{c}$.

Proof. (a) If $x \in C_{q}(f)$, then $x \notin \bar{M}_{f}$. Choose $\delta>0$ such that $(x-\delta, x+\delta) \cap$ $M_{f}=\varnothing$. Let $\varepsilon>0$ be given. Since $x \in C_{q}(f)$, there is a neighborhood $I$ of $x$ with $|I|<\delta$ and a set $S$ residual in $I$ such that $|f(y)-f(x)|<\varepsilon$ for $y \in S$. From this it is clear that

$$
\left|q-\varlimsup_{y \rightarrow t} f(y)-f(x)\right| \leqslant \varepsilon \quad \text { for } t \in I .
$$

Since $I \cap M_{f}=\varnothing$, this says that

$$
\left|\mu_{f}(t)-f(x)\right| \leqslant \varepsilon \quad \text { for } t \in I .
$$

In particular, this says that $\left|\mu_{f}(x)-f(x)\right| \leqslant \varepsilon$ and, hence, $\mu_{f}(x)=f(x)$. Restating (1), we have $\left|\mu_{f}(t)-\mu_{f}(x)\right| \leqslant \varepsilon$ for $t \in I$, and (a) is verified.

(b) Since $\mu_{f}$ agrees with $f$ on $C_{q}(f)$, and since $C_{q}(f)$ is residual, we clearly must have $\left(\mu_{f}\right)_{q}^{s}$ existing everywhere and being identical to $f_{q}^{s}$. This is simply the observation that any $g$ that agrees with $f \in \Sigma_{q}$ on a residual set will have $f_{q}^{s}$ as its $q$-symmetric derivative.

(c) If $x \notin \bar{M}_{f}$, then there is a neighborhood $I$ of $x$ such that

$$
\mu_{f}(y)=q-\varlimsup_{t \rightarrow y} f(t) \quad \text { for } y \in I .
$$

From this and the fact that $f$ and $\mu_{f}$ agree on the residual set $C_{q}(f)$ we have

$$
\mu_{f}(y)=q-\varlimsup_{t \rightarrow y} \mu_{f}(t) \quad \text { for } y \in I \text {. }
$$

Consequently,

$$
\mu_{f}(x) \leqslant \varlimsup_{y \rightarrow x} \mu_{f}(y)
$$


If strict inequality held, there would be a sequence $\left\{y_{n}\right\}$ converging to $x$ and a number $\alpha$ such that $\mu_{f}(x)<\alpha<\mu_{f}\left(y_{n}\right)$ for all $n$. Also, since $\mu_{f}(x)<\alpha$, there is a neighborhood $J \subseteq I$ of $x$ such that

$$
\{y: f(y)<\alpha\} \text { is residual in } J .
$$

Pick $n$ so large that $y_{n} \in J$. Since $\mu_{f}\left(y_{n}\right)>\alpha$,

$$
\{y: f(y)>\alpha\} \text { is second category in } J,
$$

and the inconsistency of (2) and (3) completes the proof of part (c).

(d) Let $\alpha \in R$ and let $\left\{I_{n}\right\}$ be an enumeration of the intervals contiguous to $\bar{M}_{f}$. From part (c) we have that the restriction of $\mu_{f}$ to any $I_{n}$ is a function of Baire class one on $I_{n}$. Consequently,

$$
\left\{x: \mu_{f}(x)>\alpha\right\}=\left[\bigcup_{n}\left\{x \in I_{n}: \mu_{f}(x)>\alpha\right\}\right] \cup\left\{x \in \bar{M}_{f}: \mu_{f}(x)>\alpha\right\}
$$

is clearly an $F_{\sigma}$ set. Similarly, $\left\{x: \mu_{f}(x)<\alpha\right\}$ is an $F_{\sigma}$. Hence, $\mu_{f} \in \beta_{1}$.

(e) Let $x_{0} \in\left(\bar{M}_{f}\right)^{c}$. We first consider the case where $f_{q}^{s}\left(x_{0}\right)$ is finite. Without loss of generality, we may assume $x_{0}=0=f_{q}^{s}(0)$. Given $\varepsilon>0$, there is a $\delta>0$ such that $(-\delta, \delta) \cap M_{f}=\varnothing$ and a residual subset $S$ of $(0, \delta)$ such that

$$
f(t)-f(-t)<2 t \varepsilon \quad \text { for } t \in S .
$$

Since $C_{q}(f)$ is residual, we may assume $S \subseteq C_{q}(f)$.

Fix a $t \in(0, \delta)$ and choose a sequence $\left\{s_{n}\right\} \subseteq S$ such that $s_{n} \rightarrow t$ and $f\left(s_{n}\right) \rightarrow \mu_{f}(t)$. Such a sequence exists because $S$ is residual in $(0, t)$. Since $s_{n} \in C_{q}(f)$, there is a $\rho_{n}$, $0<\rho_{n}<\delta-s_{n}$ and a set $S_{n}$ residual in $\left(s_{n}, s_{n}+\rho_{n}\right)$ such that

$$
\left|f\left(s_{n}\right)-f(y)\right|<1 / n \text { for } y \in S_{n} .
$$

We may clearly choose $\rho_{n}$ so that the sequence $\left\{\rho_{n}\right\}$ converges to 0 .

For each $n$, let $T_{n}=R_{0}\left(S_{n}\right)$. Then $T_{n}$ is residual in $\left(-s_{n}-\rho_{n},-s_{n}\right)$. There is a residual set $U \subseteq C_{q}(f)$ such that

$$
\varlimsup_{\substack{\alpha \in U \\ \alpha \rightarrow-t}} f(\alpha)=\mu_{f}(-t)
$$

Let $-t_{n} \in T_{n} \cap U$ for each $n$. Then $t_{n} \rightarrow t, f\left(t_{n}\right) \rightarrow \mu_{f}(t)$, and

$$
\mu_{f}(-t) \geqslant \varlimsup_{n \rightarrow \infty} f\left(-t_{n}\right) \geqslant \varlimsup_{n \rightarrow \infty}\left(f\left(t_{n}\right)-2 t_{n} \varepsilon\right)=\mu_{f}(t)-2 t \varepsilon .
$$

Since $\varepsilon$ was arbitrary, we conclude that $D^{s} \mu_{f}(0) \leqslant 0$. A similar argument shows that $D_{s} \mu_{f}(0) \geqslant 0$ and therefore $\mu_{f}^{s}(0)=0$, completing the proof of part (e) under the assumption that $f_{q}^{s}\left(x_{0}\right)$ is finite.

Next, suppose $f_{q}^{s}\left(x_{0}\right)=+\infty$ and again assume $x_{0}=0$. Given $\alpha \in R$, there is a $\delta>0$ such that $(-\delta, \delta) \cap M_{f}=\varnothing$ and a set $S$ residual in $(-\delta, \delta) \cap C_{q}(f) \cap$ $R_{0}\left(C_{q}(f)\right)$ such that

$$
f(t)-f(-t)>2 t \alpha \quad \text { for } t \in S .
$$

Fix a $t \in(0, \delta)$ and choose a sequence $\left\{s_{n}\right\} \subset S \cap(-\delta, 0)$ such that $s_{n} \rightarrow-t$ and $f\left(s_{n}\right) \rightarrow \mu_{f}(-t)$. As above, we may choose a sequence $\left\{t_{n}\right\} \subset S$ such that $t_{n} \rightarrow t$, 


$$
\begin{aligned}
f\left(-t_{n}\right) \rightarrow \mu_{f}(-t) \text { and } & \varlimsup_{\lim _{n \rightarrow \infty}} f\left(t_{n}\right) \leqslant \mu_{f}(t) . \text { Then } \\
\mu_{f}(t)-\mu_{f}(-t) & \geqslant \varlimsup_{n \rightarrow \infty} f\left(t_{n}\right)-\lim _{n \rightarrow \infty} f\left(-t_{n}\right) \geqslant \varlimsup_{n \rightarrow \infty}\left(f\left(t_{n}\right)-f\left(-t_{n}\right)\right) \\
& \geqslant \lim _{n \rightarrow \infty} 2 t_{n} \alpha=2 t \alpha .
\end{aligned}
$$

Since $\alpha$ is arbitrary, this implies $\mu_{f}^{s}(0)=+\infty$, and this completes the proof for the case where $f_{q}^{s}\left(x_{0}\right)=+\infty$. A similar argument handles the case $f_{q}^{s}\left(x_{0}\right)=-\infty$.

An examination of the proof of part (e) in the previous theorem shows that a little more can be said in the case where $M_{f}$ has no limit points.

THEOREM 4.5. If $f \in \Sigma_{q}$ and $M_{f}$ has no limit points, then there is a residual set $S$ and $a g \in \Sigma$ such that $f_{q}^{s}(x)=g^{s}(x)$ for all $x$ and $f(x)=g(x)$ for $x \in S$.

Proof. Note that if $x_{0} \in R$, there is a $\delta>0$ such that $\left(x_{0}-\delta, x_{0}+\delta\right) \cap$ $\left[M_{f} \backslash\left\{x_{0}\right\}\right]=\varnothing$ and the same argument as employed in Theorem 4.4(e) may be employed to show that $f_{q}^{s}\left(x_{0}\right)=\left(\mu_{f}\right)^{s}\left(x_{0}\right)$. Simply let $g=\mu_{f}$ and $S=C_{q}(f)$.

THEOREM 4.6. If $f \in \Sigma_{q}$, then $\left|\left\{x:\left|f_{q}^{s}(x)\right|=\infty\right\}\right|=0$.

Proof. First, recall that the symmetric derivative of a measurable function can be infinite only on a set of measure zero [9]. Apply this result to $\mu_{f}$ on each component interval of $\left(\bar{M}_{f}\right)^{c}$, recalling that $\left(\mu_{f}\right)^{s}=f_{q}^{s}$ there.

Next, we look for a monotonicity theorem and examine some consequences. The notion of a qualitative symmetric cover will be used here.

Definition 4.3. For each $x \in R$, let $\delta(x)$ be a positive-valued function and let $N_{x}$ be a first category set. Define

$$
J_{x}=\left\{[x-\delta, x+\delta]: \delta<\delta(x) \text { and }\{x-\delta, x+\delta\} \subseteq\left(N_{x}\right)^{c}\right\}
$$

and $J=\cup_{x \in R} J_{x}$. We call $J$ a qualitative symmetric cover for $R$.

LEMMA 4.2. Let $J$ be a qualitative symmetric cover for $R$ and let $x_{0} \in R$. If

$P=\left\{\delta>0: J\right.$ contains a partition of $\left.\left[x_{0}-\delta, x_{0}+\delta\right]\right\}$,

then $P$ is residual in $(0, \infty)$.

Proof. Without loss of generality we may assume $x_{0}=0, \delta(x)$ is even, and $N_{x}=N_{-x}$. Let

$\rho=\sup \left\{\delta: P^{c} \cap(0, \delta)\right.$ is a first category set $\}$.

Clearly $\rho \geqslant \delta(0)>0$. Suppose $\rho<\infty$. Then for every $\varepsilon>0$ the set $P^{c} \cap(\rho, \rho+\varepsilon)$ is of second category. But this is clearly impossible if $0<\varepsilon<\delta(\rho)$. Thus, $\rho=\infty$.

THEOREM 4.7. If $f \in \Sigma_{q}$ and $f_{q}^{s} \geqslant 0$ everywhere, then $f$ is nondecreasing on $C_{q}(f)$.

Proof. Let $\varepsilon>0$ be given. For $x \in R$, there is a $\delta(x)>0$ and a first category set $N_{x} \subset(x-\delta(x), x+\delta(x))$ such that whenever $0<\delta<\delta(x)$ and $\{x-\delta, x+\delta\} \subseteq$ $\left(N_{x}\right)^{c}$

$$
f(x+\delta)-f(x-\delta)>-2 \varepsilon \delta
$$


This is so since $f_{q}^{s}(x) \geqslant 0$. Let $J_{x}$ be the collection of all such intervals $[x-\delta, x+\delta]$ and $J=\cup J_{x}$. Then $J$ is a qualitative symmetric cover for $R$.

Let $a<b$ be two points in $C_{q}(f)$ and let $x_{0}=(a+b) / 2$. For any $\delta>0$ for which $J$ contains a partition of $\left[x_{0}-\delta, x_{0}+\delta\right]$, we clearly have

$$
f\left(x_{0}+\delta\right)-f\left(x_{0}-\delta\right)>-2 \varepsilon \delta .
$$

From the lemma we have that the collection of all such $\delta$ 's is residual in $(0, \infty)$, and since $\{a, b\} \subseteq C_{q}(f)$, it follows that

$$
f(b)-f(a) \geqslant-(b-a) \varepsilon,
$$

and since $\varepsilon$ is arbitrary, $f(b) \geqslant f(a)$.

This method of deducing monotonicity theorems from covering theorems was developed by B. Thomson in [19], where he applied it to ordinary, approximate, and symmetric derivatives.

THEOREM 4.8. If $f \in \Sigma_{q}$ and $f_{q}^{s}$ is bounded above or bounded below, then there is a residual set $S$ and $a g \in \Sigma$ such that $f_{q}^{s} \equiv g^{s}$ and $\left.f\right|_{S}=\left.g\right|_{S}$.

Proof. Suppose $f_{q}^{s}$ is bounded below. We lose no generality in assuming that $f_{q}^{s}$ is bounded below by 0 . Then the previous theorem guarantees that $f$ is nondecreasing on the residual set $C_{q}(f)$ and hence $M_{f}=\varnothing$ and Theorem 4.5 can be applied.

\section{REFERENCES}

1. C. L. Belna, G. T. Cargo, M. J. Evans and P. D. Humke, Analogues of the Denjoy-Young-Saks Theorem, Proc. Amer. Math. Soc. 271 (1982), 253-260.

2. C. L. Belna, M. J. Evans and P. D. Humke, Symmetric and ordinary differentiation, Proc. Amer. Math. Soc. 72 (1978), 261-267.

3. Symmetric monotonicity, Acta Math. Acad. Sci. Hungar. 34 (1979), 17-22.

4. J. C. Burkill and U. S. Haslam-Jones, The derivates and approximate derivates of measurable functions, Proc. London Math. Soc. (2) 32 (1930), 346-355.

5. S. Chow, On approximate derivatives, Bull. Amer. Math. Soc. 54 (1948), 793-802.

6. E. P. Dolženko, Boundary properties of arbitrary functions, Math. USSR-Izv. 1 (1967), 1-12.

7. M. J. Evans and P. D. Humke, Equality of unilateral derivates, Proc. Amer. Maih. Soc. 79 (1980), $609-613$

8. A Khintchine, Recherches sur la structure des fonctions mesurables, Rec. Math. Soc. Math. Moscow 31 (1924), 265-285 and 377-433.

9. Recherches sur la structure des fonctions mesurables, Fund. Math. 9 (1927), 212-279.

10. L. Larson, The Baire class of approximate symmetric derivates, Proc. Amer. Math. Soc. 87 (1983), $125-130$.

11. The symmetric derivative, Trans. Amer. Math. Soc. 277 (1983), 589-599.

12. J. L. Leonard, Some conditions implying the monotonicity of a real function, Rev. Roumaine Math. Pures Appl. 12 (1972), 757-780.

13. S. Marcus, Sur la limite approximative qualitative, Com. Acad. R. P. Române 3 (1953), 9-12. (Roumanian; French summary)

14. __ Sur la continuité approximative qualitative, Com. Acad. R. P. Române (1953), 117-120. (Roumanian; French summary)

15. ___ Sur la dérivée approximative qualitative, Com. Acad. R. P. Române (1953), 361-364. (Roumanian; French summary)

16. C. J. Neugebauer, $A$ theorem on derivates, Acta Sci. Math. (Szeged) 23 (1962), 79-81.

17. J. C. Oxtoby, Measure and category, Springer-Verlag, New York, Heidelberg, and Berlin, 1971.

18. S. Saks, Theory of the integral, Dover, New York, 1964.

19. B. Thomson, On full covering properties, Real Anal. Exchange 6 (1980), 77-93. 
20. L. Zajiček, Sets of $\sigma$-porosity and sets of $\sigma$-porosity ( $q$ ), Časopis Pěst. Mat. 101 (1976), 350-359.

2i. On the symmetry of Dini derivates of arbitrary functions, Comment. Math. Univ. Carolin. 22 (1981), 195-209.

22. A. Zygmund, Trigonometric series, 2nd ed., Cambridge Univ. Press, Cambridge, 1959.

Department of Mathematics, North Carolina State University, Raleigh, North Carolina 27650 (Current address of M. J. Evans)

Department of MATHEMatics, WeStern Illinois University, Macomb, Illinois 61455

Current address (L. Larson): Department of Mathematics, University of Louisville, Louisville, Kentucky 40292 